\title{
ASPECTOS DA REPRODUÇÃO DE PALAEMON PANDALIFORMIS (STIMPSON) (DECAPODA, PALAEMONIDAE) NO MANGUEZAL DE RATONES, FLORIANÓPOLIS, SANTA CATARINA
}

\author{
Yara Maria Rauh Müller ${ }^{1}$ \\ Evelise Maria Nazari ${ }^{1}$ \\ Cristine Maria Bressan ${ }^{1}$ \\ Dib Ammar 1,2
}

\begin{abstract}
REPRODUCTIVE ASPECTS OF PALAEMON PANDALIFORMIS (STIMPSON) (Decapoda, Palaemonidae) at Ratones Mangrove, Florianópolis, Santa CATARINA. Biometric and reproductive aspects of Palaemon pandaliformis (Stimpson, 1871) from Ratones Mangrove, Florianópolis, Santa Catarina, have been investigated. Individuals were monthly collected from August, 91 to July, 93 and the temperature and salinity registered. Biometry, sex-ratio, sexual maturity, fecundity and reproductive cycle have been determined. The temperature showed defined temporal distribution. This species presents annual reproductive cycle, relatively high individual and populational fecundity, and the ovigerous females were larger than males and other females of the same species.

KEY WORDS. Reproduction, Palaemon pandaliformis, ratones mangrove, fecundity
\end{abstract}

Palaemon pandaliformis (Stimpson, 1871), palemonídeo que habita águas doce e salgada, é encontrado na América, desde a Guatemala até o sul do Brasil (Holthuis 1952; 1980). Segundo Bond-Buckup \& Buckup (1989) P. pandaliformis é a única espécie do gênero que ocorre no Brasil meridional, tendo sido citada para os Estados do sul do Brasil por KRETZSCHMAR (1984) e Müller et al. (1990). Na Ilha de Santa Catarina é um gênero representativo de palemonídeos, encontrado preferencialmente nos manguezais (MÜLLER \& FERREIRA 1992).

Este palemonídeo é registrado em águas onde a temperatura varia de 18 a $21^{\circ} \mathrm{C}$, em ambiente completamente doce ou, no auge da estação seca, em salinidades próximas às do mar, sendo capturados com frequência junto às raízes da vegetação submersa (Coelho 1963; Coelho \& Ramos-Porto 1985; Bond-Buckup \& BuCKuP 1989; Coelho \& Coelho-SANTOS 1990).

Palaemon pandaliformis é uma espécie de pequeno porte que praticamente não é utilizada na dieta alimentar de populações humanas, mas serve como iscas em pescarias. Popularmente em algumas regiões do nordeste brasileiro é conhecido como potitinga (HolTHUIS 1980).

1) Departamento de Biologia Celular, Embriologia e Genética, Universidade Federal de Santa Catarina. Caixa Postal 476, 88040-900 Florianópolis, Santa Catarina, Brasil.

2) Bolsista de Iniciação à Pesquisa (DAP/UFSC). 
São poucas as informações disponíveis na literatura sobre aspectos da reprodução desta espécie. CoELHo (1963) relata, para o litoral de Pernambuco, a ocorrência de fêmeas ovígeras nos meses de abril, maio, junho, julho e outubro e, que a fecundidade individual de uma fềmea de $14,00 \mathrm{~mm}$ de comprimento de carapaça é de aproximadamente 290 ovos. GOMES-CORRÊA (1977) analisou fềmeas ovígeras, provenientes de Estados da região sudeste, com comprimento total de 26,00 a 40,00mm que apresentavam numerosos ovos. KRETZSCHMAR (1984) afirma ter a fêmea tamanho similar ao apresentado pelo macho e relata a ocorrência de fêmeas ovígeras em capturas efetuadas no litoral do Estado do Paraná, nos meses de janeiro, abril e dezembro. J. Araújo (comunicação pessoal) observou, para a Ilha de Santa Catarina, fêmeas ovígeras com comprimento total de 34,60 a $45,30 \mathrm{~mm}$ e peso de 0,15 a $0,87 \mathrm{~g}$, cuja fecundidade individual oscilava entre 54 e 183 ovos, estando representadas nos meses de janeiro, março, abril, novembro e dezembro. Estudos complementares sobre a reprodução de $P$. pandaliformis fornecerão informações importantes para o melhor conhecimento da biologia reprodutiva desta espécie.

\section{MATERIAL E MÉTODOS}

Palaemon pandaliformis foi coletada mensalmente, no período de agosto de 1991 a julho de 1993, em um canal no Manguezal de Ratones, Ilha de Santa Catarina, localizado aproximadamente a $27^{\circ} 28^{\prime} \mathrm{S}$ e $48^{\circ} 29^{\prime} \mathrm{W}$. Os animais foram capturados, com um puçá de malha de $0,5 \mathrm{~cm}$, por entre a vegetação submersa das margens e no fundo do canal. Foram registrados dados relativos à temperatura do ar, da água e salinidade.

As fêmeas ovígeras, distinguidas dos demais exemplares por apresentar câmara incubadora contendo ovos, foram fixadas em bouin alcoólico e os demais exemplares em formol a 10\%. Após 24 horas o material foi transferido para álcool a $70 \%$ a partir do qual foram realizados estudos biométrico e reprodutivo.

Procedeu-se então a determinação do comprimento total (distância entre as extremidades distais do rostro e do télson) e a pesagem dos indivíduos. Os valores obtidos foram organizados em sete classes, com intervalos de $5 \mathrm{~mm}$ e $0,13 \mathrm{~g}$, respectivamente.

A maturidade sexual individual foi determinada pelo comprimento total apresentado pela menor fêmea ovígera capturada. A estimativa da maturidade sexual populacional foi feita determinando-se o intervalo de comprimento total correspondente à classe de comprimento em que se completa $50 \%$ do total de fêmeas constituintes da população.

O ciclo reprodutivo foi estabelecido com base nos períodos de ocorrência de fêmeas ovígeras ao longo dos meses de estudo.

Os ovos foram foram retirados das fêmeas ovígeras com o auxílio de pinça de ponta fina e estiletes histológicos, após o que foram contados com registrador manual. Os valores registrados por fêmea foram organizados em 6 classes, com intervalos de 100 ovos. 
A estimativa da fecundidade da espécie foi estabelecida através de correlações entre os parâmetros: fecundidade/comprimento total e fecundidade/peso total. O modelo empregado foi o da regressão linear, através do método dos mínimos quadrados, com a equação $Y=a+b X$, ao nível de significância de $p \leq 0,05$, através do programa estatístico Statgraphics versão 5.0.

\section{RESULTADOS}

Palaemon pandaliformis foi capturado no Manguezal de Ratones sempre nas margens do canal e abrigado entre as raizes da vegetação submersa, em condições ambientais onde a temperatura do ar variou de 15 a $33^{\circ} \mathrm{C}$ e a temperatura da água de 16 a $31^{\circ} \mathrm{C}$. A quantidade de sais dissolvidos na água foi de 14 a $32 \%$, indicando que o local de coleta é um ponto que sofre bastante influência da maré.

Capturou-se em 17 coletas, 222 exemplares sendo 84 machos (38\%), 138 fêmeas (62\%), apresentando razão sexual de 0,6 . Verificou-se em 11 coletas a ocorrência de 53 fêmeas ovígeras, perfazendo $24 \%$ do total de indivíduos amostrados (Fig. 1). Estas fêmeas foram registradas, principalmente nos meses de dezembro de 1991 a março de 1992 e dezembro de 1992 a maio de 1993, definindo dois períodos reprodutivos.

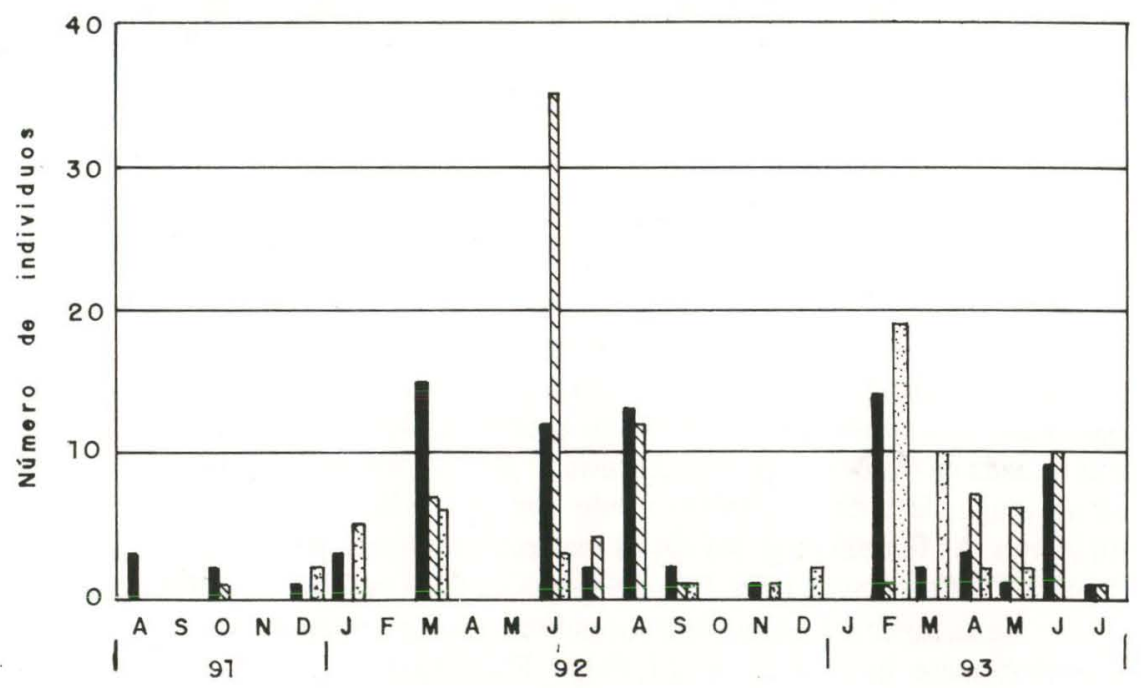

Machos Fêmeas Fèmeas Ovigeras

Fig. 1. Distribuição de machos, fêmeas e fêmeas ovígeras de $P$. pandaliformis no Manguezal de Ratones no periodo de 08/91 a 07/93.

Com relação à variável peso, observou-se que os machos oscilavam entre 0,04 e $0,51 \mathrm{~g}$, as fêmeas entre 0,05 e $0,70 \mathrm{~g}$ e as fêmeas ovigeras entre $0,21 \mathrm{e} 0,91 \mathrm{~g}$. A distribuição dos indivíduos em classes de peso (Fig. 2) mostra que os machos 
estão concentrados nas três classes iniciais e a maioria deles tem peso superior a $0,13 \mathrm{~g}$ e inferior a $0,39 \mathrm{~g}$. As fêmeas apresentam peso mais elevado, encontrando-se principalmente nas faixas compreendidas entre 0,26 e $0,65 \mathrm{~g}$. As fêmeas ovígeras são os indivíduos de maior peso e estão distribuídos principalmente nas classes de 0,39 a $0,78 \mathrm{~g}$.

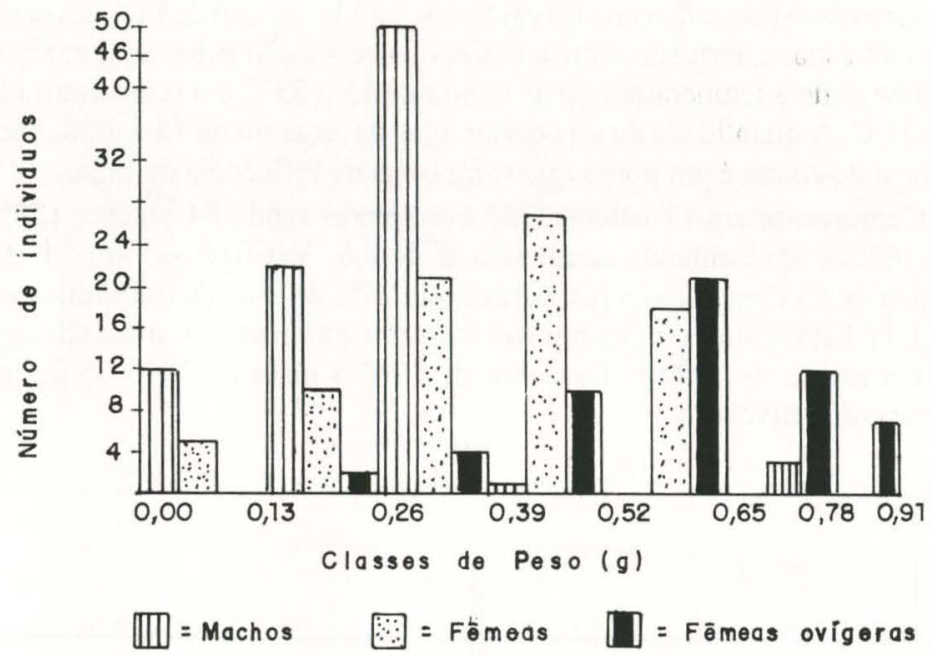

Fig. 2. Distribuição em classes de peso de Palaemon pandaliformis no Manguezal de Ratones no periodo de $08 / 91$ a $07 / 93$.

Os machos mediam de 20,30 a 41,80 mm, as fềmeas de 16,00 a $48,00 \mathrm{~mm}$ e as fêmeas ovígeras de 27,40 a $50,40 \mathrm{~mm}$. A figura 3 mostra a distribuição dos indivíduos em classes de comprimento total onde, $61 \%$ da população de machos está situada na classe compreendida entre 31,00 a $36,00 \mathrm{~mm}$. As fêmeas distribuemse ao longo de todas as classes sendo que $88 \%$ delas situam-se entre 31,00 e $46,00 \mathrm{~mm}$. As fêmeas ovígeras são os maiores indivíduos, presentes nas classes de 26,00 a $51,00 \mathrm{~mm}$, sendo que $57 \%$ delas está na faixa de 41,00 a $46,00 \mathrm{~mm}$.

Nesta espécie a maturidade sexual individual é obtida quando a fêmea atinge o comprimento aproximado de $27,40 \mathrm{~mm}$. Da análise dos dados de comprimento total relativos a todas as fêmeas, verifica-se que a maturidade sexual populacional ocorre quando elas atingem a classe de 36,00 a $41,00 \mathrm{~mm}$ e, com um comprimento médio de $38,50 \mathrm{~mm}$ todas estão adultas e aptas à reprodução.

O número de ovos carregados pelas fềmeas variou de 107 a 684 , sendo a média individual de 420,15. A maioria das fêmeas ovígeras (77\%) carrega entre 300 e 700 ovos em sua câmara incubadora (Fig. 4).

Se comparados os valores médios do número de ovos carregados por fêmea, distribuídos em classes de comprimento (Tab. I), pode-se observar que as fêmeas 
ovígeras de comprimento menor carregam menos ovos porém, quando comparadas entre si, as classes de $36,00-41,00 \mathrm{~mm}$ e a de $41,00-46,00 \mathrm{~mm}$, nota-se que, nestas duas classes, é muito próximo o número de ovos transportados pelas fêmeas.

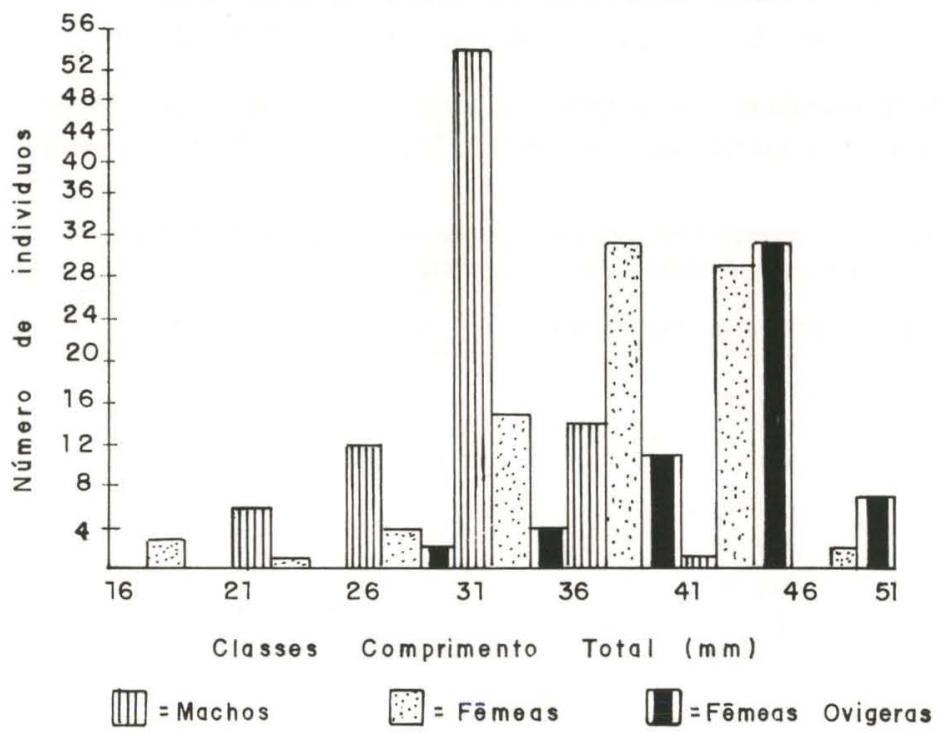

Fig. 3. Distribuição em classes de comprimento de $P$. pandaliformis no Manguezal de Ratones no no período de 08/91 a 07/93.

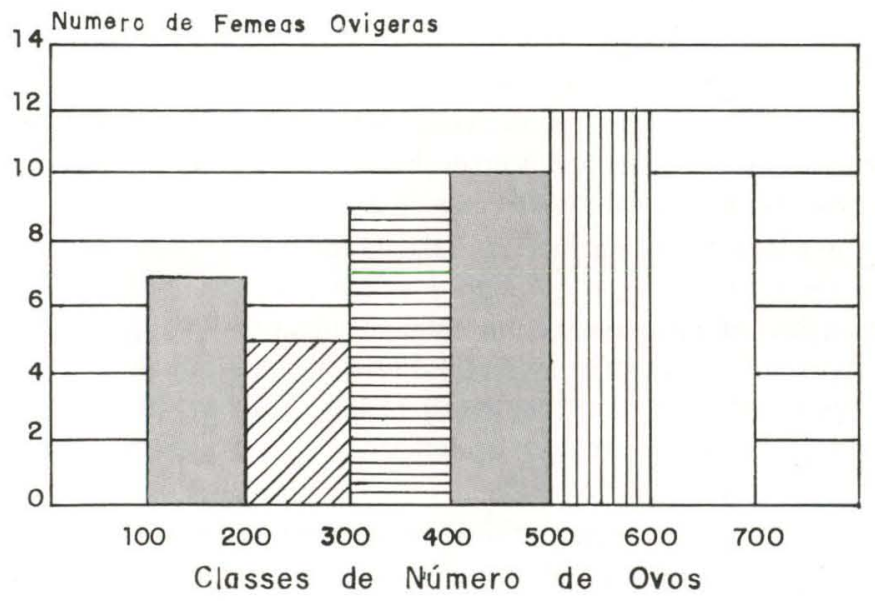

Fig. 4. Distribuição das fêmeas ovígeras de Palaemon pandaliformis em classes de número de ovos. 
Para fêmeas que apresentam comprimento total entre 27,40 e 50,40 mm (Fig. 5) e peso de 0,21 a 0,9lg (Fig. 6), obteve-se, através da relação entre as variáveis fecundidade/comprimento total e fecundidade/peso, as seguintes equações lineares:

$$
\begin{aligned}
& F=-125.603+12.8117 L, \text { com } r=0,35 e^{2} r^{2}=12,44 \\
& F=335.07+107.746 W, \text { com } r=0,10 e r^{2}=1,03
\end{aligned}
$$

onde: (F) fecundidade, $(\mathbf{L})$ comprimento total em milímetros, $(\mathbf{W})$ peso em gramas, (r) coeficiente de correlação de Pearson, $\left(\mathbf{r}^{2}\right)$ coeficiente de determinação.

Tabela I. Número médio de ovos correspondente às classes de comprimento total de fêmeas ovigeras de Palaemon pandaliformis.

\begin{tabular}{cc}
\hline Classe de comprimento total $(\mathrm{mm})$ & Número médio de ovos \\
\hline $16 \vdash 21$ & - \\
$21-26$ & - \\
$261-31$ & 154,5 \\
$31-36$ & 294,3 \\
$36-41$ & 422,3 \\
$41 \vdash-46$ & 429,8 \\
$46 \vdash 51$ & 491,6 \\
\hline
\end{tabular}

\section{DISCUSSÃO}

O ambiente estuarino no Manguezal de Ratones, no qual $P$. pandaliformis foi estudado, corresponde ao descrito na literatura por COELHO (1963), COELHO \& RAMOS-PORTO (1985), BOND-BUCKUP \& BUCKUP (1989), COELHO \& COELHOSANTOS (1990) e RAMOS-PORTO \& COELHO (1990). O pequeno número de indivíduos capturados em algumas coletas parece ter sido influenciado diretamente pelas variações no nível da água do canal, seja na baixa-mar, estiagem ou período chuvoso. Isto se deve, provavelmente, a que em períodos secos a vegetação marginal fica totalmente exposta e eles precisam deslocar-se para outros locais e, em períodos chuvosos, são facilmente carregados pela água.

De maneira geral, na família Palaemonidae, os machos são os maiores indivíduos (BosCHI 1974), o que não é válido para $P$. pandaliformis, onde os machos de maior comprimento são normalmente menores do que as menores fêmeas ovígeras. Apesar de terem sido capturados indivíduos maiores do que os citados por Gomes-CoRrÊA (1977), KRETZSCHMAR (1984) e Bond-BuCKuP \& BUCKUP (1989), principalmente em relação às fêmeas ovígeras, a espécie pode ainda ser considerada como de pequeno porte.

Os registros das temperaturas do ar e da água nos dias de coleta mostram que esta variável apresenta uma distribuição temporal bem definida. Estes palemonídeos vivem em temperaturas variadas, superando os valores considerados por COELHO \& RAMOS-PORTO (1985), para a costa brasileira, como os limites extremos de temperatura da água onde vive esta espécie. 


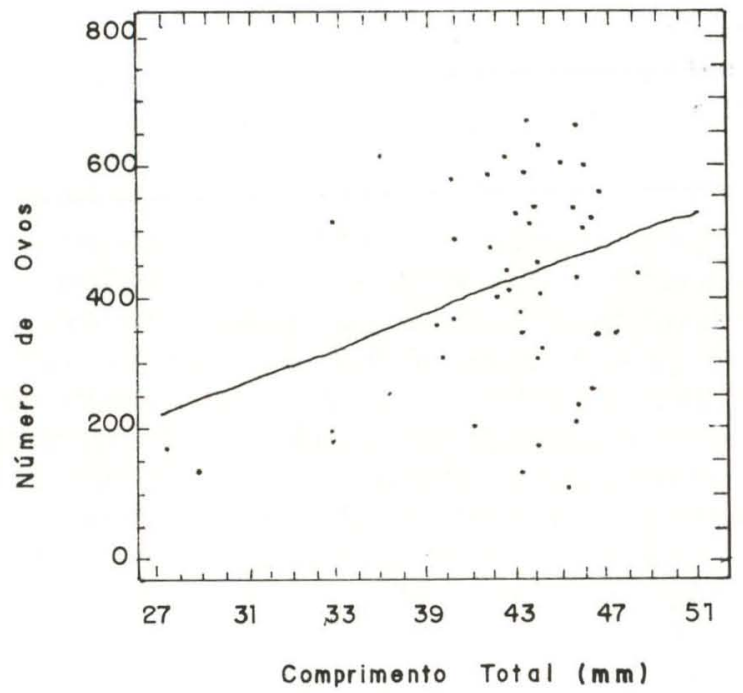

Fig. 5. Relação entre a fecundidade e o comprimento total de Palaemon pandaliformis no Manguezal de Ratones no período de 08/91 a 07/93.

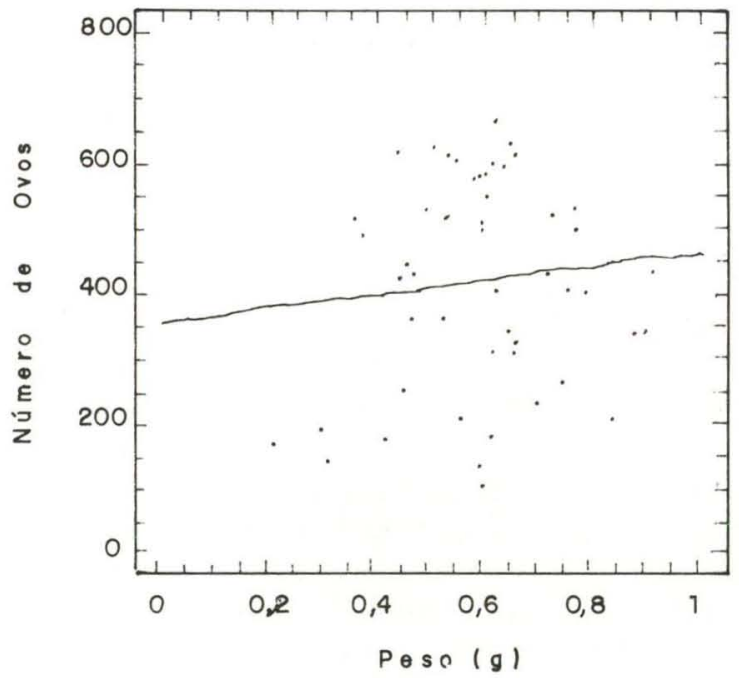

Fig. 6. Relação entre a fecundidade e o peso de Palaemon pandaliformis no Manguezal de Ratones no periodo de 09/91 a 07/93.

A captura de fêmeas ovígeras ocorreu preferencialmente nos meses em que a temperatura da água esteve mais alta. Observações feitas por J. Araújo (comunicação pessoal) em outros locais do Manguezal de Ratones confirmam a presença de fêmeas ovígeras nos meses de verão. De acordo com FiguERAS (1986), o período 
reprodutivo de outras espécies do gênero Palaemon também ocorre nos meses em que a temperatura está mais elevada.

A salinidade aparentemente não foi relevante na regulação do ciclo reprodutivo de $P$. pandaliformis, pois salinidades muito diferentes foram registradas nas duas estações reprodutivas e nenhum padrão definido de distribuição foi constatado.

Ciclos reprodutivos anuais são característicos para a família Palaemonidae em determinadas condições e ambientes (CARVALHO 1978; LowE \& PROVENZANO 1990). O período natural de desova da espécie registrado no Manguezal de Ratones corresponde, em parte, à ocorrência de fêmeas ovígeras relatadas por CoELHO (1963) e KRETZSChMAR (1984). O fato de os dois períodos reprodutivos não apresentarem a mesma amplitude, com duração maior do segundo, pode ser interpretado como um deslocamento (Boolootian et al. 1959; GIESE \& PEARSE 1974), comuns em crustáceos com reprodução anual e provavelmente estão associados ao aumento da temperatura. A ocorrência de um pequeno número de fềmeas ovígeras fora do período reprodutivo está de acordo com o esperado no processo de reprodução de palemonídeos (CARVALHO 1978).

Palaemon pandaliformis carrega um número de ovos superior ao registrado para Macrobrachium amazonicum (Heller, 1862) (MAGALHÃES 1989), Macrobrachium potiuna (Müeller, 1880) (MÜLlER \& CARPES 1991) e Palaemonetes argentinus (Nobili, 1901) (J. Araújo comunicação pessoal). Contudo os valores registrados para $P$. pandaliformis por CoelHo \& Coelho-SANTOS (1990) é inferior ao valor médio de ovos registrado no Manguezal de Ratones. O número de ovos transportados pelas fêmeas que apresentavam comprimento entre 36,00 e $46,00 \mathrm{~mm}$ é muito semelhante e próximo ao valor médio registrado, indicando que, atingido um determinado comprimento, a fêmea apresenta câmara incubadora com espaço adequado à disposição de um certo número de ovos. Isto mostra que existe um limite quanto ao potencial reprodutivo da espécie analisada.

Considerando as dimensões da espécie, a fecundidade individual e a fecundidade média populacional podem ser consideradas altas. As fêmeas que carregavam um número de ovos inferior a 300 , ou eram de pequeno porte ou os ovos estavam em etapas finais do desenvolvimento e, possivelmente, alguns já haviam eclodido.

A dispersão dos dados de fecundidade mostra que há uma correlação com tendência positiva entre as variáveis fecundidade e comprimento total, fecundidade e peso. O valor do coeficiente de correlação entre o número de ovos e o comprimento total dos indivíduos, indica que a correlação entre estas duas variáveis é regular, enquanto que para número de ovos e peso a correlação é fraca (ZAR 1974). Desta forma, o número de ovos transportados pelas fêmeas desta população parece estar pouco relacionado com o porte, devendo-se levar em conta outros fatores como a idade reprodutiva e a capacidade da gônada de produzir um maior ou menor número de gametas.

A maioria dos autores (LOBÃo et al. 1985, 1986; VALENTI et al. 1989) não utiliza o coeficiente de determinação $\left(\mathrm{r}^{2}\right)$ na estimativa de fecundidade, o que inviabiliza uma análise mais apurada. BOND \& BUCKUP (1982) analisam o valor 
deste coeficiente para Macrobrachium borelli (Nobili, 1836) e M. potiuna, onde os valores obtidos são bem superiores aos de $P$. pandaliformis.

Palaemon pandaliformis parece garantir o sucesso de seu processo reprodutivo por carregar seus ovos até a eclosão em uma câmara incubadora e encontrar no Manguezal de Ratones condições favoráveis à sua reprodução.

\section{REFERÊNCIAS BIBLIOGRÁFICAS}

Boolootian, R.A.; A.C. Giese; J. FarmaiAn \& J. TUCKer. 1959. Reproductive cycles of five west coast crabs. Phys. Zool. 32 (4): 213-220.

BonD, G. \& L. BUCKUP. 1982. O ciclo reprodutor de Macrobrachium borelli (Nobili, 1836) e Macrobrachium potiuna (Müller, 1880) (Crustacea, Decapoda, Palaemonidae) e suas relações com a temperatura. Rev. Brasil. Biol. 42 (3): 473-483.

Bond-Buckup, G. \& L. Buckup. 1989. Os Palaemonidae de Águas Continentais do Brasil Meridional (Crustacea, Decapoda). Rev. Brasil. Biol. 49 (4): 883-896. BoschI, E.E. 1974. Biologia de los crustaceos cultivables en America Latina. Carpas 06: 1-24.

CARVALHO, H.A. 1978. Ciclo sexual de Macrobrachium acanthurus (Wiegmann, 1836)-(Crustacea, Decapoda): Relações com fatores abióticos e ciclo de intermudas. Tese de Doutorado, não publicada, Universidade de São Paulo, São Paulo, 199p.

CoELHO, P.A. 1963. Algumas observações sobre a biologia e ecologia dos camarões Palaemon northropi e P. pandaliformis no Estado de Pernambuco (Decapoda-Palaemonidae). Bol. Inst. Oceanogr. Univ. Recife 5/6: 69-72.

Coelho, P.A. \& M. Ramos-Porto. 1985. Camarões de Água Doce do Brasil: Distribuição Geográfica. Revta bras. Zool. 2 (6): 405-410.

Coelho, P.A. \& M.A. Coelho-Santos. 1990. Crustáceos decápodos e estomatópodos dos rios Paripe, Itamaracá-PE. Anais Soc. Nord. Zool. 3: 35-42.

FIgUeRAS, A.J. 1986. Crecimiento de Palaemon adspersus (Rathke, 1837) y $P$. serratus (Pennant, 1777) (Decapoda: Natantia) en la ria de Vigo (SO de Espanã). Inv. Pesq. 50 (1): 117-126.

GIESE, A. C. \& J. S. PEARSE. 1974. Reproduction of marine invertebrates. New York, Academic Press, vol. 1, XV+546p.

Gomes-CorrêA, M.M. 1977. Palaemonídeos do Brasil (Crustacea, Decapoda, Natantia). Dissertação de Mestrado, não publicada, Universidade Federal do Rio de Janeiro, Rio de Janeiro, 135p.

Holthuis, L.B. 1952. A general revision of the Palaemonidae (Crustacea, Decapoda, Natantia) of the Americas. II. The subfamily Palaemoninae. Allan Hancock Foun. Publ. 12: 1-396.

1980. FAO species catalogue. 1. Shrimps and prawns of the world. FAO

Fish Synop. 125: 1-261.

Kretzschmar, S.Z. 1984. Camarões de água doce do litoral do Paraná. Dissertação de Mestrado, não publicada, Universidade Federal do Paraná, 
Curitiba, 138p.

Lobão, V.L.; W.C. Valenti \& J.T.C. Mello. 1985. Fecundidade em Macrobrachium carcinus (L.) do Rio Ribeira do Iguape. Bol. Inst. Pesca 12 (3): $1-8$.

LoBÃo, V. L.; N.E.T. RoJas \& W.C. VALENTI. 1986. Fecundidade e fertilidade de Macrobrachium amazonicum (Heller, 1862) (Crustacea, Decapoda) em laboratório. Bol. Inst. Pesca 13 (2): 15-20.

LowE, B.T. \& A.J. PRovenZANO. 1990. Survival and reproduction of Palaemonetes paludosus (Gibbes, 1850) (Decapoda: Palaemonidae) in saline water. J. Crust. Biol. 10 (4): 639-647.

MagalHãEs, C. 1989. The larval development of palaemonid shrimps from the Amazon region reared in the laboratory. VI. Abbreviated development of Macrobrachium nattereri (Heller, 1862) (Crustacea: Decapoda). Amazoniana 10 (4): 379-392.

Müller, Y.M.R.; A.C. Prazeres \& J. ARaúJo. 1990. Ocorrência de espécies da família Palaemonidae (Malacostraca, Decapoda) na Ilha de Santa Catarina-SC. Anais II Simpósio Ecossistemas da Costa Sul e Sudeste BrasileiraEstrutura, Função e Manejo. Águas de Lindóia, 2: 273-278.

MÜLLER, Y.M.R. \& S. CARPES. 1991. Macrobrachium potiuna (Müller, 1880):

Aspectos do ciclo reprodutivo e sua relação com parâmetros ambientais (Crustacea, Decapoda, Palaemonidae). Revta bras. Zool. 8 (1/2/3/4): 23-30.

Müller, Y.M. R. \& E.C. FERREIRA. 1992. Dados preliminares de palemonídeos coletados no Manguezal de Ratones-Florianópolis/SC. Anais $44^{\mathrm{a}}$ Reunião Anual SBPC, São Paulo, p.855.

Ramos-Porto, M. \& P.A. Coelho. 1990. Sinopse dos crustáceos decápodos brasileiros (Família Palaemonidae). Anais Soc. Nord. Zool. 3: 93-111.

VAlenti, W.C.; J.T.C. Mello \& V.L. Lobão. 1989. Fecundidade em Macrobrachium acanthurus (Wiegmann, 1836) do Rio Ribeira do Iguape. Revta bras. Zool. 6 (1): 9-15.

ZAR, J.H. 1974. Biostatistical Analysis. London, Prentice-Hall, XV+620p.

Recebido em 14.III.1996; aceito em 18.XI.1996. 